Revue de droit comparé du travail et de la sécurité sociale

2| 2018

Harcèlement moral au travail en jurisprudence

comparée

\title{
Reconnaissance du caractère économique de l'activité de fourniture d'assurance maladie obligatoire en Slovaquie
}

Hélène Payancé

(2) OpenEdition

Journals

Édition électronique

URL : https://journals.openedition.org/rdctss/1963

DOI : $10.4000 /$ rdctss. 1963

ISSN : 2262-9815

Éditeur

Centre de droit comparé du travail et de la sécurité sociale

Édition imprimée

Date de publication : 1 juin 2018

Pagination : 120-123

ISSN : 2117-4350

Référence électronique

Hélène Payancé, « Reconnaissance du caractère économique de l'activité de fourniture d'assurance maladie obligatoire en Slovaquie ", Revue de droit comparé du travail et de la sécurité sociale [En ligne] 2 | 2018, mis en ligne le 01 novembre 2021, consulté le 13 novembre 2021. URL : http:// journals.openedition.org/rdctss/1963; DOI : https://doi.org/10.4000/rdctss.1963

\section{(c) $(1)$}

Revue de droit comparé du travail et de la sécurité sociale est mise à disposition selon les termes de la Licence Creative Commons Attribution - Pas d'Utilisation Commerciale - Pas de Modification 4.0 International. 


\title{
RECONNAISSANCE DU CARACTÈRE ÉCONOMIQUE DE L'ACTIVITÉ DE FOURNITURE D'ASSURANCE MALADIE OBLIGATOIRE EN SLOVAQUIE
}

\author{
Affaire T-216/15 du 5 février 2018:
}

S'il existe, au niveau communautaire, une réglementation qui a pour objectif d'assurer l'effectivité de la libre circulation des travailleurs au sein de l'Union européenne, en veillant à ce qu'un travailleur ne perde pas ses droits en matière de sécurité sociale lors de l'exercice de son droit de se déplacer dans I'Union, cette réglementation ne réalise qu'une simple coordination des systèmes nationaux de sécurité sociale ${ }^{1}$. II ne s'agit pas d'une harmonisation de ceux-ci. Dès lors, chaque Etat membre est libre de déterminer son système de sécurité sociale et notamment les conditions d'affiliation à ce système ${ }^{2}$.

L'affaire présentée devant le Tribunal concerne le système de sécurité sociale de la Slovaquie. Cet Etat a fait le choix, en 1994, de passer d'un système d'assurance maladie unitaire, avec une seule société d'assurance maladie publique, à un mode mixte où organismes publics et privés coexistent. Tous les organismes d'assurance maladie, publics comme privés, fournissent la couverture de l'assurance maladie obligatoire aux résidents slovaques.

Parmi ces organismes, un organisme privé a déposé une plainte auprès de la Commission des Communautés européennes au sujet d'une aide d'Etat présumée qui aurait été octroyée par la République slovaque aux seuls organismes publics. La Commission a considéré, en substance, que les mesures litigieuses ne constituaient pas une aide d'Etat, au motif que l'activité d'assurance maladie obligatoire organisée et exercée en République slovaque ne saurait être considérée comme une activité économique et que, dès lors, les organismes publics, en tant que bénéficiaires de ces mesures, ne sauraient être qualifiés d'entreprises au sens de l'article 107, paragraphe 1, du TFUE.

Interrogé sur ce point, le tribunal a quant à lui considéré que la possibilité pour les sociétés d'assurance maladie de pouvoir réaliser, utiliser et distribuer une partie de leurs bénéfices est de nature à remettre en cause le caractère non économique de leur activité. II relève que la Commission a reconnu que la possibilité d'utiliser et de distribuer des

1 Les règles de coordination communautaire des législations nationales de sécurité sociale ne permettent aucunement aux personnes de choisir leur sécurité sociale parmi les différentes législations des Etats membres de I'UE. Au contraire, ces règles se limitent à préciser la « législation nationale applicable » aux différentes situations transfrontalières.

2 CJCE 24 avril 1980, aff. C-110/79, Una Coonan contre Insurance Officer. Dans un communiqué du 27 octobre 2004, la commission européenne a rappelé « que les Etats membres conservent l'entière maîtrise de l'organisation de leur système de protection sociale ; cela vaut en particulier pour toute l'étendue des dispositions légales et réglementaires concernant la sécurité sociale (article 137 du traité CE) » avant de préciser que « le marché commun des assurances complémentaires, mis en place depuis 1992, n'implique donc, en aucun cas, le renoncement aux systèmes légaux de protection sociales des Etats membres, pas plus que la modification de leur organisation ». 
bénéfices est encadrée de manière plus stricte que dans les secteurs marchands classiques, puisque cette faculté est, en l'espèce, subordonnée au respect d'exigences destinées à garantir la pérennité du régime et la réalisation des objectifs sociaux et solidaires qui le sous-tendent. Toutefois, le tribunal a estimé que ce constat est dépourvu de pertinence aux fins d'exclure la nature économique de l'activité, dès l'instant où les opérateurs du marché en cause s'inscrivent dans une logique de recherche de profits. En effet, la possibilité pour les sociétés d'assurance maladie slovaques de rechercher et de réaliser librement des bénéfices témoigne, en tout état de cause, indépendamment de l'exécution de leur mission d'assurance maladie publique et du contrôle étatique opéré, du fait qu'elles poursuivent une finalité lucrative et, partant, que les activités qu'elles exercent sur le marché relèvent de la sphère économique. Dès lors, l'existence de conditions strictes encadrant postérieurement l'utilisation et la distribution des bénéfices qui pourraient résulter de ces activités ne permet pas de remettre en cause la nature économique de ces dernières.

Par ailleurs, le tribunal a considéré que l'existence d'une certaine concurrence sur la qualité et sur l'étendue de l'offre, à laquelle se livrent les différents organismes au sein du régime slovaque d'assurance maladie obligatoire, influe également sur le caractère économique de l'activité. En effet, si les organismes d'assurance maladie ne peuvent pas librement fixer le montant des cotisations et se concurrencer formellement en matière tarifaire, le législateur a néanmoins introduit un élément de concurrence en matière de qualité, puisque les organismes peuvent librement compléter les prestations légales obligatoires par des prestations connexes gratuites, telles qu'une meilleure prise en charge de certains types de traitement complémentaire et préventif dans le cadre des prestations de base obligatoires ou l'existence d'un service renforcé d'assistance aux assurés. Ils peuvent dès lors se différencier en matière de qualité et d'étendue de l'offre pour attirer des assurés, qui, en vertu de la loi, sont libres de choisir leur société d'assurance maladie et d'en changer une fois par an. La marge de liberté dont disposent les organismes pour se concurrencer permet ainsi aux assurés de bénéficier d'une meilleure protection sociale pour un niveau de cotisation équivalant, puisque les prestations complémentaires offertes le sont à titre gratuit. Ainsi, même si les organismes d'assurance maladie slovaques sont tenus d'offrir les mêmes prestations légales, ils se font concurrence sur le « rapport qualité-prix » de la couverture qu'ils offrent et, partant, sur la qualité et l'efficacité des processus d'achat.

Ainsi, le tribunal a estimé que l'activité de fourniture d'assurance maladie obligatoire en Slovaquie présente, eu égard au but lucratif poursuivi par les sociétés d'assurance maladie et à l'existence d'une intense concurrence sur la qualité et sur l'offre de services, un caractère économique.

Ce raisonnement ne suscite pas de critique car si la concurrence présente au sein du régime slovaque ne porte ni sur les prestations légales obligatoires ni formellement sur le montant des cotisations, celle-ci ne saurait être niée compte tenu de la volatilité du marché, qui résulte de la possibilité pour les assurés de choisir librement leur prestataire d'assurance maladie et d'en changer une fois par an, et du fait qu'elle concerne la qualité du service, qui relève de l'appréciation personnelle des assurés.

La portée de cette décision mérite des précisions dans la mesure où seulement quelques jours après avoir été rendue le MLPS ${ }^{3}$ a publié sur son site internet un communiqué

3 Le Mouvement pour la Liberté de la Protection Sociale, fondé en 1991, fait partie des mouvements qui prétendent que les travailleurs indépendants ou salariés peuvent quitter la sécurité sociale française au profit d'une assurance privée européenne considérée comme moins chère et plus protectrice en s'autoproclamant « libérés de la sécu ». 
annonçant que «le tribunal européen a sonné définitivement le glas du monopole de la sécurité sociale».

Soyons clairs, l'obligation d'affiliation à la sécurité sociale en France n'a nullement été remise en cause par cette décision européenne qui concerne un pays où, à la différence de la France, les assurés ont le choix entre plusieurs organismes de sécurité sociale pour la couverture de l'assurance maladie obligatoire.

Toute personne qui travaille en France est obligatoirement affiliée à un régime de sécurité sociale français déterminé ${ }^{4}$. Il est possible, pour améliorer sa protection sociale, de bénéficier de couvertures complémentaires auprès d'entreprises d'assurance, de mutuelles ou d'institutions de prévoyance ou également d'organismes assureurs établis dans un autre Etat membre de l'Union européenne. Cependant, ces couvertures professionnelles ou individuelles ne peuvent pas se substituer à la sécurité sociale.

C'est parce que les régimes de sécurité sociale mettent en œuvre une solidarité ${ }^{5}$ que leurs organismes peuvent/doivent être soustraits du champ des règles régissant la concurrence entre les entreprises. "Les régimes de sécurité sociale opèrent une redistribution censée mettre en œuvre une solidarité entre les membres d'un groupe déterminé face à certains risques. Pour que joue cette solidarité il faut que tous les membres du groupe soient contraints de verser à un pot commun des contributions indépendantes de leur vulnérabilité personnelle au risque envisagé, ce pot commun étant affecté au financement des prestations versées aux victimes de ce risque (...). Cette solidarité suppose, par hypothèse, le pot commun dont la gestion doit, par hypothèse, être confiée à une seule et même institution ou à des institutions entre lesquelles est aménagée une compensation financière $»^{6}$.

La jurisprudence européenne a déjà eu l'occasion de vérifier la compatibilité de l'obligation de cotiser en France à la sécurité sociale avec les règles européennes.

Ainsi, dans les célèbres arrêts Poucet et Pistre 7 , la CJCE a écarté l'argumentation de deux travailleurs indépendants français qui avaient cessé de cotiser à la sécurité sociale pour souscrire uniquement une assurance privée en soutenant que les caisses de sécurité sociale seraient des entreprises au sens des articles 85 et 86 , en situation de monopole ou d'abus de position dominante. La Cour rejeta l'application du droit de la concurrence au litige en jugeant que les régimes de sécurité sociale «poursuivent un objectif social et obéissent au principe de solidarité », les caisses et organismes qui en assurent la gestion " concourent à la gestion du service public de la sécurité sociale », ils « remplissent une fonction de caractère exclusivement social » et ne sont dès lors pas des entreprises car leur activité est « fondée sur le principe de la solidarité nationale et dépourvue de tout but lucratif ».

Par ailleurs, la CJCE, saisie au sujet du recours d'un travailleur indépendant qui avait cessé de cotiser aux régimes maladie et vieillesse de sécurité sociale français pour souscrire à la place une assurance privée, a jugé dans l'arrêt $\operatorname{Garcia}^{8}$ que les dispositions de la directive

4 Article L.111-2-2 CSS.

5 Le Préambule de la Constitution du 27 octobre 1946 reconnaît un droit pour tous à une sécurité sociale élevée et solidaire. L'article L.111-1 du Code de la sécurité sociale rappelle le principe de solidarité.

6 J.-J. DUPEYROUX, «Les exigences de la solidarité », Dr. social 1990, p. 741.

7 CJCE 17 février 1993, aff. jointes C-159/91, Poucet contre AGF et C-160/91, Pistre contre CANCAVA.

8 CJCE 26 mars 1996, aff. C-238/94, Garcia contre mutuelle de prévoyance sociale d'Aquitaine. 
92/49/CEE sur l'abolition de monopoles visent non pas les risques couverts par la sécurité sociale mais certains domaines de l'assurance privée. Dans l'arrêt Kattner Stahlbau', la Cour a précisé que les règles européennes de la libre prestation de services sont compatibles avec une obligation d'affiliation dès lors que le régime en cause ne va pas au-delà de ce qui est nécessaire pour atteindre l'objectif consistant à assurer l'équilibre financier d'une branche de la sécurité sociale.

L'obligation d'affiliation à la sécurité sociale en France n'a pas davantage été remise en question par l'arrêt de la CJUE en date du 3 octobre $2013^{10}$ selon lequel « la directive sur les pratiques commerciales déloyales (devait) être interprétée en ce sens que relève de son champ d'application personnel un organisme de droit public en charge d'une mission d'intérêt général, telle que la gestion d'un régime légal d'assurance maladie ». Dans cet arrêt, il doit être observé que la BKK, caisse allemande d'assurance maladie, menait à titre subsidiaire des opérations commerciales, et que c'est pour ce type d'opérations qu'elle était soumise au respect des dispositions de la directive 2005/29/CE du 11 mai 2005 relative aux pratiques commerciales déloyales vis-à-vis des consommateurs dans le marché intérieur. La Cour de cassation, dans un arrêt du 18 juin 201511, a confirmé que le recouvrement de contraintes de sécurité sociale dues par une personne assujettie à titre obligatoire au régime de protection sociale des travailleurs non-salariés ne revêtait pas le caractère d'une pratique commerciale au sens des dispositions de l'article 2, d) de la directive 2005/29/CE du Parlement européen et du Conseil du 11 mai 2005 relatives aux pratiques déloyales, et n'entrait donc pas dans le champ d'application de cette directive. Cette analyse ne méconnaît pas la décision de la CJUE précitée, mais en précise la portée en la restituant dans le contexte de la directive, à savoir l'interdiction des pratiques déloyales vis-à-vis des consommateurs, y compris du fait d'un organisme de sécurité sociale qui se livre, parallèlement à son activité d'intérêt général, à des pratiques commerciales ${ }^{12}$.

Espérons que le jugement rendu par le tribunal correctionnel de Paris le 22 février 2018 condamnant quatre personnes et deux associations, dont le MLPS, à des peines de prison avec sursis et des amendes pour « incitation au refus des assujettis de se conformer aux prescriptions de la législation de sécurité sociale ${ }^{13}$ sonne enfin le glas de ces campagnes de désinformation visant à favoriser le remplacement d'un système solidaire de sécurité sociale par la mise en place d'un système de libre choix d'assurance privée avec tarification individualisée.

9 CJUE 5 mars 2009, aff. C-350/07, Kattner Stahlbau contre Maschinenbau.

10 CJUE 3 octobre 2013, aff. C-59/12, BKK contre Zentrale zur Bekämpfung unlauteren Wettbewerbs.

11 Pourvoi n 14-18049.

12 Pour des illustrations récentes de l'ensemble des questions relatives à la compatibilité avec les règles européennes, voir les jugements du 20 septembre 2016 et du 24 janvier 2017 du TASS de la Gironde.

13 Un des prévenus a été condamné à dix mois d'emprisonnement avec sursis et son association à 60000 euros d'amende pour moitié avec sursis. Un autre prévenu a été condamné à cinq mois d'emprisonnement avec sursis. Une autre association a été condamnée à 10000 euros d'amende dont 8000 avec sursis, et deux de ses dirigeants à quatre et un mois d'emprisonnement avec sursis. Notons que les sanctions prévues par l'article L.114-18 du livre 1 « dispositions communes à tout ou partie des régimes de base » du Code de la sécurité sociale, créé par la loi du 21 décembre 2006, ont été considérablement alourdies par la loi du 22 décembre 2014 pour tenter de mettre fin à la fronde. Observons, au passage, que l'article L.615-1 du livre 6 « dispositions applicables aux travailleurs indépendants " prévoit des sanctions différentes (comparez l'alinéa 1 de l'article L.114-18 et l'alinéa 2 de l'article L.615-1). 